\title{
Hybrid Simulated Annealing-Tabu Search Method for Optimal Sizing of Autonomous Power Systems With Renewables
}

\author{
Yiannis A. Katsigiannis, Pavlos S. Georgilakis, Senior Member, IEEE, and Emmanuel S. Karapidakis, Member, IEEE
}

\begin{abstract}
Small autonomous power systems (SAPS) that include renewable energy sources are a promising option for isolated power generation at remote locations. The optimal sizing problem of SAPS is a challenging combinatorial optimization problem, and its solution may prove a very time-consuming process. This paper initially investigates the performance of two popular metaheuristic methods, namely, simulated annealing (SA) and tabu search (TS), for the solution of SAPS optimal sizing problem. Moreover, this paper proposes a hybrid SA-TS method that combines the advantages of each one of the above-mentioned metaheuristic methods. The proposed method has been successfully applied to design an SAPS in Chania region, Greece. In the study, the objective function is the minimization of SAPS cost of energy $(€ / \mathrm{kWh})$, and the design variables are: 1) wind turbines size, 2) photovoltaics size, 3) diesel generator size, 4) biodiesel generator size, 5) fuel cells size, 6) batteries size, 7) converter size, and 8) dispatch strategy. The performance of the proposed hybrid optimization methodology is studied for a large number of alternative scenarios via sensitivity analysis, and the conclusion is that the proposed hybrid SA-TS improves the obtained solutions, in terms of quality and convergence, compared to the solutions provided by individual SA or individual TS methods.
\end{abstract}

Index Terms-Hybrid power systems, optimal sizing, optimization methods, power generation dispatch, renewable energy sources, simulated annealing (SA), small autonomous power systems (SAPS), solar energy, tabu search (TS), wind energy.

\section{INTRODUCTION}

$\mathbf{T}$ ODAY more diverse challenges have emerged: climate change, economic recession, and security of energy supply. Moreover, the rapid depletion of fossil fuels and their high and volatile prices have necessitated an urgent need for alternative energy sources to meet the energy demands [1]. Renewable energy sources (RES), such as wind and solar, are clean, inexhaustible, and environmentally friendly alternative energy sources with negligible fuel cost. However, RES technologies, such as wind turbines (WTs) and solar photovoltaics (PVs), are dependent on a resource that is unpredictable and depends on weather and climatic changes, and the production

Manuscript received July 14, 2011; revised November 21, 2011; accepted January 12, 2012. Date of publication April 06, 2012; date of current version June 15, 2012.

Y. A. Katsigiannis and E. S. Karapidakis are with the Department of Natural Resources and Environment, Technological Educational Institute of Crete, Chania 73133, Greece (e-mail: karapidakis@chania.teicrete.gr).

P. S. Georgilakis is with the School of Electrical and Computer Engineering, National Technical University of Athens (NTUA), Athens 15780, Greece (e-mail: pgeorg@power.ece.ntua.gr).

Digital Object Identifier 10.1109/TSTE.2012.2184840 of WTs and PVs may not match with the load demand, so there is an impact on the reliability of the electric energy system. This reliability problem can be solved by a proper combination of the two resources (WTs and PVs) together with the use of an energy storage system, such as batteries, as a type of energy-balancing medium [2]. Such a system, which is called a small autonomous power system (SAPS), is a promising option for isolated power generation at remote locations [3]. To be more precise, an SAPS is an isolated hybrid system with renewable energy sources, conventional power sources (usually diesel generators), and energy storage. Proper sizing of the overall SAPS is challenging, due to the large number of design options and the uncertainty in key parameters, such as load size and future fuel price. Renewable energy sources add further complexity because their power output is unpredictable and intermittent.

The problem of SAPS optimal sizing belongs to the category of combinatorial optimization problems, since the sizes of system's components, which constitute the design variables, can take only discrete values. For the solution of this problem, various deterministic optimization techniques have been proposed [4]; however, these methods may provide suboptimal solutions, which are usually combined with increased computational complexity. The most direct method for solving the SAPS sizing problem is the complete enumeration method that is used by HOMER software [5]; however, it can prove to be extremely time consuming. Moreover, a recent review of computer tools has shown that there is no energy tool that addresses all issues related to SAPS optimal sizing, but instead the "ideal" energy tool highly depends on the specific objectives that must be fulfilled [6].

In recent years, new methods have been developed, in order to solve many types of complex optimization problems, particularly those of combinatorial nature. These methods are called metaheuristics and include genetic algorithms (GAs), simulated annealing (SA), tabu search (TS), and particle swarm optimization (PSO) among others. Metaheuristics orchestrate an interaction between local improvement procedures and higher-level strategies to create a process capable of escaping from local optima and performing a robust search of a solution space. From the area of metaheuristics, GAs [7]-[9], SA [10], TS [11], as well as PSO [12], have been proposed for the solution of SAPS optimal sizing.

This paper initially investigates the application of two metaheuristic methods, namely SA and TS, for solving the SAPS sizing problem. SA transposes the technique of annealing to the 
solution of an optimization problem. TS is a powerful iterative optimization procedure that is characterized by its ability to escape from local optima (which usually cause conventional algorithms to terminate) by using a flexible memory system. Moreover, this paper proposes a hybrid optimization methodology that combines the above-mentioned methods (SA and TS) for solving the SAPS sizing problem. Hybrid methods that contain SA and TS have been applied in various areas, including unit commitment [13], optimal capacitor placement [14], and nonpermutation flowshop scheduling [15]. The proposed method has been successfully applied to design an SAPS in the Chania region, Greece. In the study, the objective function is the minimization of SAPS cost of energy $(€ / \mathrm{kWh})$, and the design variables are: 1) WTs size, 2) PVs size, 3) diesel generator size, 4) biodiesel generator size, 5) fuel cells size, 6) batteries size, 7) converter size, and 8) dispatch strategy. The performance of the proposed hybrid SA-TS optimization methodology is studied for a large number of alternative scenarios, and it is concluded that it improves the obtained solutions, in terms of quality and convergence, compared to the solutions provided by individual SA or individual TS method.

\section{Problem Formulation}

The SAPS optimal sizing problem has to fulfil the objective defined by (1) subject to the constraints (3)-(9). The computations of the objective function and the constraints of the problem are related with the results obtained by simulating the operation of SAPS for a given time step $\Delta t$, taking into account components' type, cost, and technical characteristics.

\section{A. Objective Function}

Minimization of the system's cost of energy (COE)

$$
\min (\mathrm{COE}) \text {. }
$$

The $\mathrm{COE}(€ / \mathrm{kWh})$ of SAPS is calculated as follows:

$$
\mathrm{COE}=\frac{C_{\text {antot }}}{E_{\text {anloadserved }}}
$$

where $C_{\text {antot }}(€)$ is the total annualized cost and $E_{\text {anloadserved }}$ $(\mathrm{kWh})$ is the total annual useful electric energy production. $C_{\text {antot }}$ takes into account the annualized capital costs, the annualized replacement costs, the annual operation and maintenance (O\&M) costs, and the annual fuel costs (if applicable) of the system's components. COE is adopted since it is a very good measure of system cost for SAPS sizing [16].

\section{B. Constraints}

1) Initial cost constraint

$$
\mathrm{IC} \leq \mathrm{IC}_{\max }
$$

where IC $(€)$ is the initial installation cost of the system, and $\mathrm{IC}_{\max }(€)$ is the maximum acceptable initial cost of the system.
2) Unmet load constraint

$$
f_{\mathrm{UL}}=\frac{\sum_{\Delta t}^{\text {year }} \mathrm{UL}_{\Delta t} \cdot \Delta t}{E_{\text {anload }}} \leq f_{\mathrm{UL} \max }
$$

where $f_{\mathrm{UL}}$ is the annual unmet load fraction, $\mathrm{UL}_{\Delta t}(\mathrm{~kW})$ is the unmet load during the simulation time step $\Delta t(\mathrm{~h})$, $E_{\text {anload }}(\mathrm{kWh})$ is the total annual electric energy demand, and $f_{\mathrm{UL} \text { max }}$ is the maximum allowable annual unmet load fraction. In this paper, $\Delta t=10 \mathrm{~min}$ so 52560 summations are needed for the entire year to compute $f_{\mathrm{UL}}$, as (4) implies.

3) Capacity shortage constraint

$$
f_{\mathrm{CS}}=\frac{\sum_{\Delta t}^{\text {year }} \mathrm{CS}_{\Delta t} \cdot \Delta t}{E_{\text {anload }}} \leq f_{\mathrm{CS} \max }
$$

where $f_{\mathrm{CS}}$ is the annual capacity shortage fraction, $\mathrm{CS}_{\Delta t}$ $(\mathrm{kW})$ is the capacity shortage during $\Delta t$, and $f_{\mathrm{CS} \text { max }}$ is the maximum allowable annual capacity shortage fraction. Capacity shortage is defined as a shortfall that occurs between the required amount of operating capacity (load plus required operating reserve) and the actual operating capacity the system can provide. Operating reserve in an SAPS with RES technologies is the surplus electrical generation capacity (above that required to meet the current electric load) that is operating and is able to respond instantly to a sudden increase in the electric load or a sudden decrease in the renewable power output.

4) Fuel consumption constraint

$$
\sum_{\Delta t}^{\text {year }} \mathrm{FCo}_{\text {gen } \Delta t} \leq \mathrm{FCo}_{\text {angen max }}
$$

where $\mathrm{FCo}_{\mathrm{gen} \Delta t}$ is the fuel consumption of a generator during $\Delta t$, and $\mathrm{FCo}_{\text {angen max }}$ is the maximum allowable annual fuel consumption of the generator.

5) Minimum renewable fraction constraint

$f_{\text {RES }}=\frac{E_{\text {anRES }}}{E_{\text {antot }}} \geq f_{\text {RES min }}$ where $0 \leq f_{\text {RES min }} \leq 1$

where $f_{\text {RES }}$ is the RES fraction of the system, $E_{\text {anRES }}$ $(\mathrm{kWh})$ is the total annual renewable energy production, $E_{\text {antot }}(\mathrm{kWh})$ is the total annual energy production of the system, and $f_{\text {RES min }}$ is the minimum allowable RES fraction.

6) Components' size constraints

$$
\begin{aligned}
& \text { size }_{\text {comp }} \geq 0 \quad \forall \quad \text { comp } \\
& \text { size }_{\text {comp }} \leq \text { size }_{\text {comp max }} \quad \forall \quad \text { comp }
\end{aligned}
$$

where size $_{\text {comp }}$ is the size of system's component comp, and size comp max $_{\text {max }}$ is maximum allowable size of comp.

\section{SAPS COMPONENTS AND MODELING}

The considered SAPS has to serve electrical load, and it can contain seven component types:

1) WTs.

2) Amorphous silicon (a-Si) PVs. 
3) Generator with diesel fuel.

4) Generator with biodiesel fuel.

5) Fuel cells (FCs) combined with reformer, using methanol as a fuel.

6) Lead-acid batteries.

7) Converter.

The modeling of SAPS components is implemented as follows. The WT modeling is implemented using a power curve profile that is based on Fuhrländer's FL 30 model. The selected WT has the following characteristics: rated power $30-\mathrm{kW} \mathrm{AC}$, cut-in speed $\left(V_{\text {in }}\right) 3 \mathrm{~m} / \mathrm{s}$, and cut-out speed $\left(V_{\text {out }}\right) 25 \mathrm{~m} / \mathrm{s}$. For the WT power curve fitting, a ninth-order polynomial expression has been selected, as it provides accurate correlation with real data, while it presents exclusively positive values for the generated power in the interval $\left[V_{\text {in }} V_{\text {out }}\right]$.

In the PV modeling, the output of the PV array $P_{\mathrm{PV}}$ (in $\mathrm{kW}$ ) is calculated from [17]

$$
P_{\mathrm{PV}}=f_{\mathrm{PV}} \cdot P_{\mathrm{STC}} \cdot \frac{G_{A}}{G_{\mathrm{STC}}} \cdot\left(1+\left(T_{C}-T_{\mathrm{STC}}\right) \cdot C_{T}\right)
$$

where $f_{\mathrm{PV}}$ is the $\mathrm{PV}$ derating factor, $P_{\mathrm{STC}}$ is the nominal $\mathrm{PV}$ array power in $\mathrm{kW}_{p}$ under standard test conditions (STCs), $G_{A}$ is the global solar radiation incident on the PV array in $\mathrm{kW} / \mathrm{m}^{2}, G_{\mathrm{STC}}$ is the solar radiation under STC $\left(1 \mathrm{~kW} / \mathrm{m}^{2}\right)$, $T_{C}$ is the temperature of the PV cells, $T_{\mathrm{STC}}$ is the STC temperature $\left(25^{\circ} \mathrm{C}\right)$, and $C_{T}$ is the $\mathrm{PV}$ temperature coefficient $\left(-0.0011 /{ }^{\circ} \mathrm{C}\right.$ for $\left.\mathrm{a}-\mathrm{Si}\right)$. The $\mathrm{PV}$ derating factor is a scaling factor applied to the PV array output to account for losses, such as dust cover, aging and unreliability of the PV array, and is considered to be equal to $0.80 . T_{C}$ can be estimated from the ambient temperature $T_{a}$ (in ${ }^{\circ} \mathrm{C}$ ) and the global solar radiation on a horizontal plane $G$ (in $\mathrm{kW} / \mathrm{m}^{2}$ ) using (11) [18]

$$
T_{C}=T_{a}+\frac{(\mathrm{NOCT}-20)}{0.8} \cdot G
$$

where NOCT is the normal operating cell temperature, which is usually obtaining the value of $48^{\circ} \mathrm{C}$.

The diesel generator fuel consumption $F(\mathrm{~L} / \mathrm{kWh})$ is assumed to be a linear function of its electrical power output [19]

$$
F=0.08415 \cdot P_{\text {rated }}+0.246 \cdot P
$$

where $P_{\text {rated }}$ is generator's rated power and $P$ is generator's output power. When biodiesel is used instead of diesel, fuel consumption is increased [20]. In this paper, a $10 \%$ increase in fuel consumption has been considered. Moreover, a 30\% minimum allowable load ratio of $P_{\text {rated }}$ has been assumed for each type of generator. Methanol has been selected as FC fuel because it presents economic, environmental, and reliability advantages for autonomous power systems [21], while the overall FC efficiency has been considered $50 \%$. In the three types of controllable generators (diesel, biodiesel, and FCs), lifetime before replacement depends on the total number of their operating hours, as calculated by the simulation process.

Lead-acid batteries have been modeled as devices capable of storing a certain amount of dc electricity at fixed round-trip energy efficiency, with limits on how quickly they can be charged or discharged, how deeply they can be discharged without causing damage, and how much energy they can cycle before needing replacement. Batteries have been modeled according to the following technical characteristics per component: capacity $1000 \mathrm{Ah}$, voltage $6 \mathrm{~V}$, round-trip efficiency 80\%, maximum charge and discharge current equal to $C / 5$, minimum state of charge $20 \%$, and lifetime throughput $9600 \mathrm{kWh}$. Finally, converter efficiency has been taken equal to $90 \%$.

The simulation process examines a particular system configuration, in which components sizes satisfy constraints (8) and (9). The necessary inputs for the simulation are: 1) annual time series data for wind speed, solar radiation, ambient temperature, and load, 2) component characteristics, 3) constraint bounds, and 4) general parameters (project lifetime, discount rate). The specific values for these data are described in Section V-A. In the simulation, for every time step $\Delta t$, the available renewable power (from WTs and PVs) is calculated and then is compared with the load. In case of excess, the surplus renewable energy is charging the batteries, if they are not fully charged. If renewable power sources are not capable to fully serve the load, the remaining electric load has to be supplied by controllable generators and/or batteries. From all possible combinations, it is selected the one that supplies the load at the least cost. When the whole year's simulation has been completed, it is determined whether the system is feasible, i.e., it is checked if it satisfies the constraints (3)-(7). After the end of simulation, the life-cycle cost of the system is calculated by taking into account: 1) the annual results of the simulation, 2) the capital, replacement, O\&M, and fuel cost (if applicable) of each component, 3) the components' lifetime, 4) the project lifetime, and 5) the discount rate. Having computed the life-cycle cost, the total annualized cost $C_{\text {antot }}$ can be calculated, which represents the hypothetical annual cost value that if it occurred each year of the SAPS lifetime, it would yield a net present cost equivalent to the actual life-cycle cost [16]. The total annual useful electric energy production $E_{\text {anloadserved }}$ that is also needed for the calculation of COE (2) is provided by simulation's results.

An additional aspect of system operation arises, which is whether (and how) the controllable generators should charge the battery bank. Two common control strategies that can be used are the load following (LF) strategy and the cycle charging (CC) strategy. It has been found [22] that over a wide range of conditions, the better of these two strategies is virtually as cost-effective as an ideal predictive strategy, which assumes the existence of perfect knowledge in future load and wind conditions. In the LF strategy, batteries are charged exclusively by nondispatchable RES technologies (WTs and PVs), whereas controllable generators (diesel, biodiesel, or FCs) produce only enough power to meet the load, if needed. An exception may occur if the required load is less than the minimum power of the operating controllable generators (due to minimum allowable load ratio constraint); then the excess generated power is stored in the batteries. LF strategy tends to be optimal in systems with a lot of renewable power, when the renewable power output sometimes exceeds the load. In the CC strategy, whenever the controllable generators need to operate to serve the load, they operate at full output power in order to achieve their maximum efficiency at their rated (maximum) power [see (12)]. A setpoint state of charge, $\mathrm{SOC}_{a}$, has also to be set in this strategy. The charging of the battery by the controllable generators 
will not stop until it reaches the specified $\mathrm{SOC}_{a}$. The battery, however, can be charged further by the nondispatchable RES technologies (WTs and PVs), if $\mathrm{SOC}_{a}<100 \%$. In this paper, three alternative values of $\mathrm{SOC}_{a}$ have been considered: $80 \%$, $90 \%$, and $100 \%$, so the total number of examined dispatch strategies is 4 . The CC strategy tends to be optimal in systems with little or no renewable power. In such systems, controllable generators produce the largest portion of power output. In order to increase the overall efficiency of the system, it is better for these generators to operate at their maximum efficiency (rated power), even if the battery losses are taken into account, rather than operating with significantly less efficiency (power) in order to meet the load at each time step.

\section{Proposed Methodology}

\section{A. Overview of Simulated Annealing}

In physics, annealing refers to the process of heating up a solid to a high temperature followed by slow cooling achieved by decreasing the temperature of the environment in steps. In the SA algorithm, the Metropolis algorithm [23] is utilized for simulating the evolution of a physical system at a given temperature $T$. By repeatedly observing this Metropolis rule of acceptance, a sequence of configurations is generated, which constitutes a Markov chain.

A finite time implementation of the SA algorithm can be realized by generating homogeneous Markov chains of finite length for a finite sequence of descending values of $T$. To achieve this, a set of parameters that governs the convergence of the algorithm has to be specified. These parameters form a cooling schedule. The parameters of the cooling schedule are:

1) The initial temperature $T_{\text {in }}$.

2) The length of the homogeneous Markov chains $L_{\text {Markov }}$.

3) The law of decrease of $T$.

4) The criterion for program termination.

From the above parameters, the law of decrease is the one that draws the most attention. The geometrical law of decrease is a widely accepted one

$$
T_{k+1}=a \cdot T_{k}
$$

where $T_{k+1}$ and $T_{k}$ are the temperatures at the $k+1$ and $k$ iteration of the algorithm, respectively, while $a$ is constant $(a<1)$. Typical values for $a$ lie between 0.5 and 0.99 [23]. An alternative solution consists of resorting to an adaptive law of the form [23]

$$
T_{k+1}=\frac{T_{k}}{1+\frac{T_{k} \cdot \ln (1+\delta)}{3 \cdot \sigma_{T k}}}
$$

where $\sigma_{T k}$ is the standard deviation of the values generated at the $k$ th Markov chain, and $\delta$ is a constant called distance parameter. Small $\delta$-values lead to small decrements in $T_{k}$. Typical values of $\delta$ are between 0.1 and 1 .

\section{B. Proposed Simulated Annealing for SAPS Optimal Sizing}

The proposed SA methodology for the optimal sizing of SAPS is composed of the following steps:

1) Setting of the parameters of the cooling schedule.
2) Random generation of an initial solution and calculation of its energy.

3) If equilibrium is achieved, go to Step 6; otherwise, repeat Steps 4 and 5.

4) Finding of a trial solution that is a neighbor to the current solution of the algorithm, and calculation of its energy. The trial solution is generated by changing randomly the value of an SAPS parameter (component size or dispatch strategy) in the current solution.

5) Performing of the acceptance test according to Metropolis algorithm.

6) If the stopping criterion is satisfied, the algorithm stops; otherwise, $T$ is decreased.

The calculation of the energy of a solution depends on its feasibility. If the solution is feasible, its energy is considered equal to the value of the objective function (COE). On the other hand, if the solution does not satisfy the constraints of the problem, the corresponding value of $\mathrm{COE}$ is considered equal to a marginal value $\mathrm{COE}_{\mathrm{marg}}$, which has to be greater than any obtained value of $\mathrm{COE}$, while at the same time it has to remain at the same order of magnitude. In this paper, $\mathrm{COE}_{\text {marg }}$ is set equal to $1 € / \mathrm{kWh}$. The total energy of a nonfeasible solution is then obtained by summing the value of $\mathrm{COE}_{\text {marg }}$ with the overall normalized penalty function.

\section{Overview of Tabu Search}

TS is a powerful optimization procedure that has been successfully applied to a number of combinatorial problems. It uses an operation called move to define the neighborhood of any given solution. TS can be viewed as an iterative technique that explores a set of problem solutions by repeatedly making moves from one solution to another, in the manner of a greatest-descent algorithm [23]. TS is characterized by the ability to escape from local optima and the occurrence of cycles, which usually cause simple descent algorithms to terminate. This goal is obtained by using a finite-size list of forbidden moves, called tabu moves, derived from the recent history of the search.

The two main components of the TS are the tabu list restrictions and the aspiration criteria of the solution associated with these restrictions. Tabu lists are managed by recording moves in the order in which they are made. If a new attribute enters into the tabu list, the oldest one is released from the tabu list. The proper choice of the tabu list size is critical to the success of the algorithm and it depends on the specific problem.

Aspiration criteria can override tabu restrictions. That is, if a certain move is forbidden, the aspiration criteria, when satisfied, can reactivate this move. The most widely used aspiration criterion removes a tabu classification from a trial move when a move yields a solution better than the best obtained so far. However, other aspiration criteria have been also proposed [23].

\section{Proposed Tabu Search for SAPS Optimal Sizing}

In the proposed TS methodology for SAPS optimal sizing, the neighborhood of a current solution contains all configurations of similar component sizes, as well as the alternative dispatch strategies options. More specifically, a move is defined by selecting each time the next larger size (if permitted) and the previous smaller size (if permitted) of a component size, while for 
the dispatch strategy a move is defined by examining the three remaining options of the current strategy. Since the SAPS contains seven components (Section III), at maximum 14 configurations with different component sizes are considered that are added to the three remaining dispatch strategies; consequently the maximum number of configurations that belong to the neighborhood of current solution is 17 .

The TS algorithm is composed of the following steps:

1) Setting of the tabu list size.

2) Generation of an initial feasible solution, and calculation of its $\mathrm{COE}$.

3) Setting of the global best solution equal to the initial solution (current solution).

4) Finding of a set of feasible trial solutions that are neighbors to the current solution and sorting of them in ascending order of $\mathrm{COE}$.

5) Checking if the selected move of the first trial solution belongs to the tabu list. If it belongs and the aspiration criterion (Step 6) is not satisfied, a selection of the next solution of the sorted set of trial solutions has to be done. Otherwise, the solution is accepted (current solution) and the update of the tabu list is performed by adding in it the chosen move, and by removing from it the oldest move, with respect to tabu list size.

6) Examination of the aspiration criterion. In the proposed algorithm, a move aspiration is satisfied if the move yields a solution better than the best obtained so far.

7) Update of the global best solution if the best acceptable solution found from the trial set has a lower COE value.

8) Repeat Steps 4-7. Stop the procedure if the termination criterion is satisfied. In this paper, the search is terminated if a maximum predefined allowable number of iterations is reached.

\section{E. Proposed Hybrid Simulated Annealing-Tabu Search for SAPS Optimal Sizing}

Hybrid optimization methods combine the advantages of individual optimization methods in order to find the optimal solution in a fast and effective manner. SA is a stochastic method that excels at gravitating towards the global optimum. However, SA is not especially fast at finding the optimum in a given solution region. For this reason, SA is often combined with local search. More specifically, SA is utilized to find the region of the optimum, and then the local optimizer takes over to find the optimum. During the local search procedure, the quality of the initial solution is essential for its successful implementation. Then the local search method is proceeding iteratively from one solution to another until a chosen termination criterion is satisfied.

This paper proposes a hybrid SA-TS optimization methodology for the solution of the SAPS sizing problem. In this methodology, SA provides the initial solution. Moreover, in order to improve the quality of results, in this paper, the conventional local search method has been replaced by TS. TS can be seen as an extension of local search, as its inherent adaptive memory ensures that the search will not return periodically and stack to the same solutions.
TABLE I

Constraint VALues For CASE Study System

\begin{tabular}{l|c|c}
\hline Constraint & Parameter & Value \\
\hline Initial cost & $I C_{\max }$ & $800,000 €$ \\
Unmet load & $f_{\text {ULmax }}$ & $1.0 \%$ \\
Capacity shortage & $f_{\text {CSmax }}$ & $2.0 \%$ \\
Fuel availability (diesel) & $F C O_{\text {angenmax }}$ & No constraint \\
Fuel availability (biodiesel) & $F C_{\text {angenmax }}$ & $40,000 \mathrm{~L} / \mathrm{y}$ \\
Fuel availability (methanol) & $F C o_{\text {angenmax }}$ & $30,000 \mathrm{~L} / \mathrm{y}$ \\
Renewable fraction & $f_{\text {RESmin }}$ & $50 \%$ \\
\hline
\end{tabular}

\section{RESUlts AND Discussion}

\section{A. Case Study System}

In the considered SAPS, the project lifetime and the discount rate are assumed to be 25 years and $5 \%$, respectively. The simulation time step $\Delta t$ is taken equal to $10 \mathrm{~min}(1 / 6 \mathrm{~h})$. The annual wind, solar, and ambient temperature data needed for the estimation of WT and PV performance refer to measurements of the Technological Educational Institute of Crete for the mountainous region of Keramia (altitude $500 \mathrm{~m}$ ), in Chania, Crete, Greece. The annual SAPS peak load has been considered equal to $120 \mathrm{~kW}$, whereas the necessary SAPS load profile was computed by downscaling the actual annual load profile of Crete island, which is the largest autonomous power system of Greece, with 600-MW peak load and 17\% min/max annual load. An additional noise has been added in the load profile, in order to reduce the $\mathrm{min} / \mathrm{max}$ annual load ratio from 17\% (Crete power system) to $12 \%$ (SAPS).

The WT hub height has been considered $25 \mathrm{~m}$, and the PVs do not include the tracking system. The operating reserve inputs, needed for the calculation of system's capacity shortage, have been considered as $5 \%$ of the average $10-\mathrm{min}$ load, $30 \%$ of the average $10-\mathrm{min}$ WT output, and $15 \%$ of the average $10-\mathrm{min}$ PV output. The values of parameters involved in constraints (3)-(7) are shown in Table I.

The cost, lifetime, and size characteristics for each component are presented in Table II. For each component, the minimum size is equal to zero. Moreover, with the exception of diesel and biodiesel generators, all components have constant increment of their size, as Table II shows. The considered sizes for the generators are $0,5,10,20,30,50,80,100$, and $120 \mathrm{~kW}$. For the SAPS sizing problem of Table II, the complete enumeration method requires

$$
\underbrace{10}_{\text {WTs }} \cdot \underbrace{41}_{\text {PVs }} \cdot \underbrace{9}_{\text {Dsl }} \cdot \underbrace{9}_{\text {Bio }} \cdot \underbrace{11}_{\text {FCs }} \cdot \underbrace{51}_{\text {Bat. }} \cdot \underbrace{51}_{\text {Conv. }} \cdot \underbrace{4}_{\text {Disp. }} \approx 3.8 \cdot 10^{9}
$$

i.e., approximately $3.8 \times 10^{9}$ evaluations in order to find the optimal COE (in (15), Disp. denotes the number of dispatch strategies). The computational time for each $\mathrm{COE}$ evaluation is 3 s. Consequently, the evaluations of the complete enumeration method require approximately 362 years. That is why it is essential to develop alternative optimization methods to solve the SAPS sizing problem in a fast and effective way.

\section{B. Simulated Annealing}

In the proposed SA algorithm, $T_{\text {in }}$ has been set equal to 2 . The termination criterion is satisfied either if $T$ reaches $10^{-5}$, 
TABLE II

COMPONENT CHARACTERISTICS

\begin{tabular}{|c|c|c|c|c|c|c|c|}
\hline Component & size $_{\text {compmax }}$ & Increment & Capital cost & Replacement cost & O\&M cost & Fuel cost & Lifetime \\
\hline WTs (30kW rated) & $9 \mathrm{WT}$ & $1 \mathrm{WT}$ & $70,000 € / \mathrm{WT}$ & $60,000 € / \mathrm{WT}$ & $500 € / y$ & - & $20 y$ \\
\hline PVs & $80 \mathrm{~kW}_{\mathrm{p}}$ & $2 \mathrm{~kW}_{\mathrm{p}}$ & $3,000 € / \mathrm{kW}_{\mathrm{p}}$ & $2,000 € / \mathrm{kW}_{\mathrm{p}}$ & 0 & - & $25 y$ \\
\hline Diesel generator & $120 \mathrm{~kW}$ & Variable & $300 € / \mathrm{kW}$ & $300 € / \mathrm{kW}$ & $0.01 € / \mathrm{h}$ per $\mathrm{kW}$ & $1.3 € / \mathrm{L}($ diesel $)$ & 20,000 oper. hours \\
\hline Biodiesel generator & $120 \mathrm{~kW}$ & Variable & $350 € / \mathrm{kW}$ & $350 € / \mathrm{kW}$ & $0.01 € / \mathrm{h}$ per $\mathrm{kW}$ & $1.5 € / L$ (biodiesel) & 20,000 oper. hours \\
\hline Fuel Cells & $50 \mathrm{~kW}$ & $5 \mathrm{~kW}$ & $3,500 € / \mathrm{kW}$ & $3,000 € / \mathrm{kW}$ & $0.02 € / h$ per $\mathrm{kW}$ & $2.0 € / \mathrm{L}$ (methanol) & 40,000 oper. hours \\
\hline Batteries (1000Ah, 6V) & 400 bat. & 8 bat. & $600 € /$ bat. & $600 € /$ bat. & $10 € /$ bat. & - & $9,600 \mathrm{kWh}$ \\
\hline Converter & $150 \mathrm{~kW}$ & $3 \mathrm{~kW}$ & $1,000 € / \mathrm{kW}$ & $1,000 € / \mathrm{kW}$ & 0 & - & $15 \mathrm{y}$ \\
\hline
\end{tabular}

TABLE III

COMPARISON OF SA ALGORITHM RESUltS (10 RUNS) FOR DIFFERENT LAWS OF TEMPERATURE DECREASE

\begin{tabular}{l|c|c}
\cline { 2 - 3 } & \multicolumn{2}{c}{ Law of temperature decrease } \\
\cline { 2 - 3 } & $a=0.5$ (geometrical) & $\delta=1$ (adaptive) \\
\hline Min cost $(€ / \mathrm{kWh})$ & 0.200331 & 0.196647 \\
Max cost $(€ / \mathrm{kWh})$ & 0.224845 & 0.210709 \\
Average cost $(€ / \mathrm{kWh})$ & 0.209712 & 0.201829 \\
Number of simulations & 540 & $540 \div 750$ \\
\hline
\end{tabular}

TABLE IV

Optimal SOLUTIONS OF SA ALgORITHM FOR DiFFERENT LAWS OF TEMPERATURE DECREASE

\begin{tabular}{l|c|c}
\cline { 2 - 3 } & \multicolumn{2}{c}{ Law of temperature decrease } \\
\cline { 2 - 3 } & $a=0.5$ (geometrical) & $\delta=1$ (adaptive) \\
\hline WTs & 5 & 6 \\
PVs $(\mathrm{kW})$ & 36 & 26 \\
Dsl $(\mathrm{kW})$ & 50 & 50 \\
Bio $(\mathrm{kW})$ & 5 & 5 \\
FCs $(\mathrm{kW})$ & 0 & 0 \\
Batteries & 352 & 320 \\
Converter $(\mathrm{kW})$ & 78 & 93 \\
Dispatch strategy & LF & LF \\
COE $(€ / \mathrm{kWh})$ & 0.200331 & 0.196647 \\
Number of simulations & 540 & 750 \\
\hline
\end{tabular}

or after three successive temperature stages without any new solution acceptance. The performance of the SA algorithm is checked for different values of $L_{\mathrm{Markov}}$ and for different parameters of the law of temperature decrease. It was found that the choice of $L_{\mathrm{Markov}}=30$ reduces significantly the number of simulations, without deteriorating the quality of the solution. Moreover, the optimal parameter values for the laws of temperature decrease are $a=0.5$ for the geometrical law, and $\delta=1$ for the adaptive law. The performance of the SA algorithm for different laws of temperature decrease is examined in Table III, which contains the results of 10 simulation runs. It can be concluded that for the same order of magnitude of COE evaluations, the adaptive law offers slightly better results. The variation of the number of simulations of the adaptive law is explained by the dependence of its temperature with the standard deviation of the Markov chain (14). Table IV presents the optimal solutions obtained for each law of temperature decrease, whereas Fig. 1 shows the evolution of the SA algorithm for the above-mentioned optimal solutions.

\section{Tabu Search}

In the TS algorithm, the determination of the initial feasible solution is essential for the successful implementation of the algorithm. This solution was created assuming exclusively AC

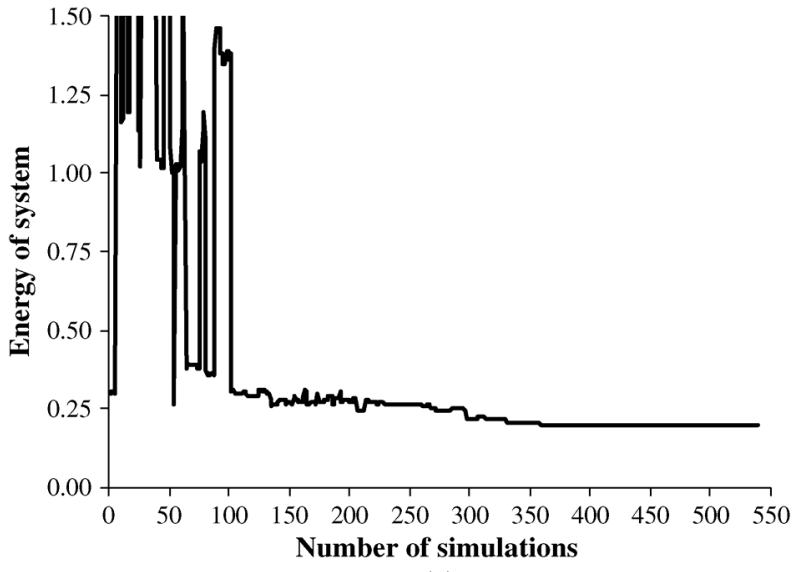

(a)

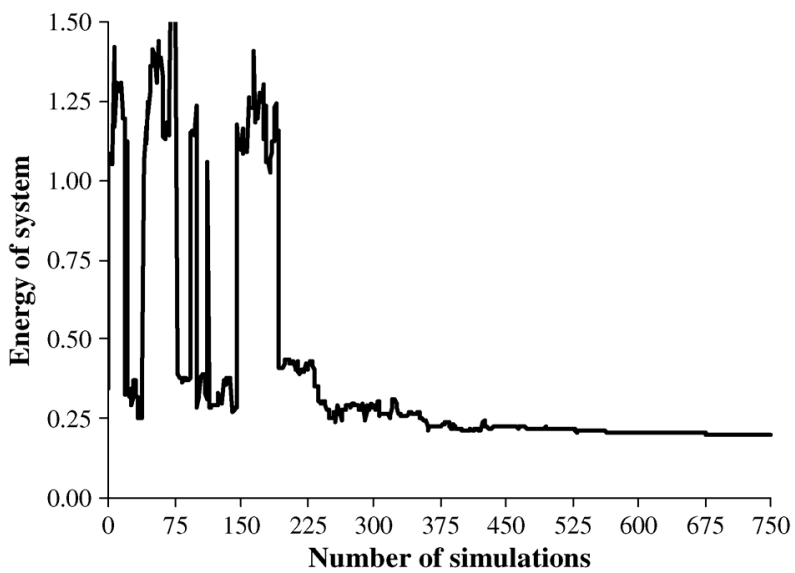

(b)

Fig. 1. Evolution of SA algorithm for different laws of temperature decrease (min cost solution): (a) geometrical law, (b) adaptive law.

components (WTs, diesel, and biodiesel generators) with large RES fraction, and it kept constant in all algorithm runs. It includes 9 WTs, a $50-\mathrm{kW}$ diesel generator, and a $120-\mathrm{kW}$ biodiesel generator, while it presents COE of $0.417005 € / \mathrm{kWh}$. The examined parameters of the TS algorithm are the number of iterations and the size of tabu list. The number of iterations was kept equal to 200 , as it provides the best solutions for all examined scenarios. The TS algorithm performance for different tabu list sizes is shown in Fig. 2, whereas Table V presents the configurations of optimal solution for different tabu list sizes. From the study of Fig. 2 and Table V, it is clear that the optimal tabu list size is 6, as smaller tabu list sizes stick in a local optimum, while larger tabu list sizes do not search thoroughly the optimal solution neighborhood. It should be noted that the adopted TS 


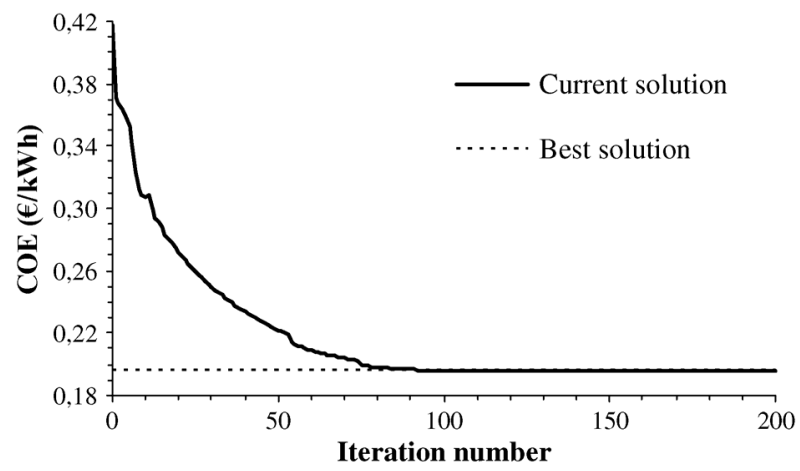

(a)

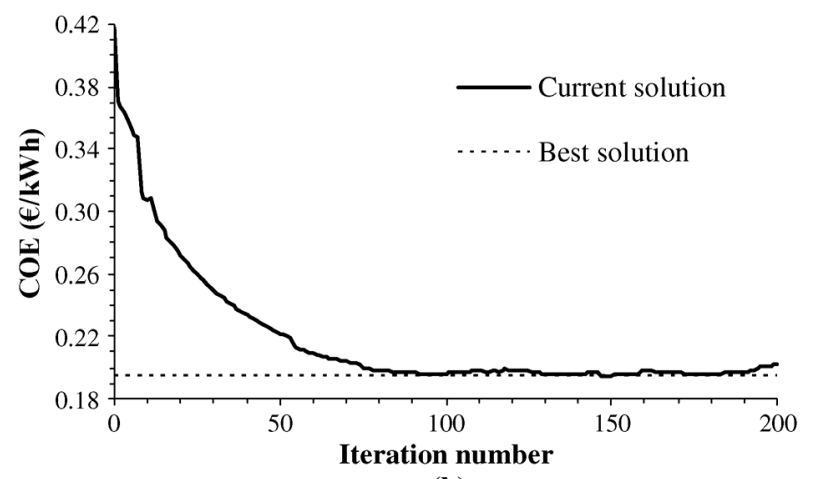

(b)

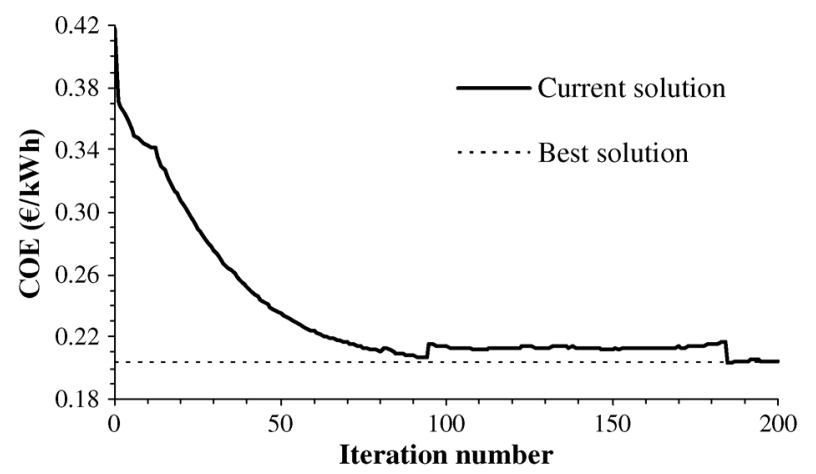

(c)

Fig. 2. Evolution of TS algorithm for different tabu list sizes: (a) tabu list size $=2$, (b) tabu list size $=6$, (c) tabu list size $=10$.

method always provides the same results, since identical initial solution and identical tabu list size have been considered. As it can be seen from Table $\mathrm{V}$, the optimal configuration contains 6 WTs, 32-kW $\mathrm{WVs}_{p}$, a 50-kW diesel generator, 304 batteries, an $84-\mathrm{kW}$ converter, LF dispatch strategy, and the COE is $0.194671 € / \mathrm{kWh}$. This COE is divided as follows: WTs $40.5 \%$, PVs $7.5 \%$, Dsl 22.3\%, Bio 0\%, FCs $0 \%$, batteries $20.7 \%$, and converter $9 \%$. The total number of performed simulations was 3708 for tabu list size equal to 6 .

\section{Hybrid Method}

From the study of Tables IV and V, it is obvious that both examined metaheuristic methods present results of similar quality, as well as significantly reduced computational burden compared to the complete enumeration method. Among the two methods, TS presents the lowest COE due to the existence of an inherent local search mechanism, which guarantees the calculation of optimal solution in a given neighborhood of solutions. On the other
TABLE V

Optimal Solutions of TS Algorithm FOR DifFERENT TABU List Sizes

\begin{tabular}{l|c|c|c}
\cline { 2 - 4 } & \multicolumn{3}{|c}{ Tabu list size } \\
\cline { 2 - 4 } & 2 & 6 & 10 \\
\hline WTs & 6 & 6 & 5 \\
PVs $(\mathrm{kW})$ & 24 & 32 & 66 \\
Dsl $(\mathrm{kW})$ & 50 & 50 & 50 \\
Bio $(\mathrm{kW})$ & 5 & 0 & 5 \\
FCs $(\mathrm{kW})$ & 0 & 0 & 0 \\
Batteries & 336 & 304 & 216 \\
Converter $(\mathrm{kW})$ & 87 & 84 & 96 \\
Dispatch strategy & LF & LF & LF \\
COE $(€ / \mathrm{kWh})$ & 0.196175 & 0.194671 & 0.203858 \\
Number of TS iterations & 95 & 147 & 185 \\
Number of simulations & 3731 & 3708 & 3530 \\
for 200 TS iterations & \multicolumn{3}{|c}{}
\end{tabular}

TABLE VI

COMPARISON OF SA (ADAPTIVE LAW-10 Runs) With Hybrid SA-TS

\begin{tabular}{l|c|c}
\cline { 2 - 3 } & \multicolumn{2}{c}{ Method } \\
\cline { 2 - 3 } & SA (adaptive) & Hybrid SA - TS \\
\hline Min cost $(€ / \mathrm{kWh})$ & 0.196647 & 0.194671 \\
Max cost $(€ / \mathrm{kWh})$ & 0.210709 & 0.208297 \\
Average cost $(€ / \mathrm{kWh})$ & 0.201829 & 0.196962 \\
Number of simulations & $540 \div 750$ & $920 \div 1130$ \\
Success rate $(\%)$ & 0 & 80 \\
\hline
\end{tabular}

hand, the TS disadvantages are the comparatively larger number of performed simulations as well as the fact that the algorithm needs to start from a feasible solution. The SA algorithm overcomes these disadvantages, but there is no guarantee that the final solution is optimal compared to its neighboring solutions.

In order to eliminate the drawbacks of individual metaheuristics (SA and TS) as well as to fully exploit their advantages, a hybrid optimization methodology that combines the above SA and TS is proposed. More specifically, the application of the TS algorithm is proposed, using as the initial (feasible) solution the obtained result from SA methodology. The performance of the hybrid method using the solutions of the 10 simulation runs of SA adaptive law is examined in Table VI. The results show the improvement of the final solution, without significantly increasing the number of required simulations. The success rate of the proposed hybrid SA-TS is $80 \%$, that is, 8 times out of 10 simulation runs the same optimal answer (i.e., $0.194671 € / \mathrm{kWh}$ minimum cost of energy) is obtained. The best solution was found only by the hybrid SA-TS, as Table VI shows; that is why the success rate is zero for SA. It should be mentioned that the application of TS requires significantly larger computational time, i.e., 3708 simulations (Table V), instead of only $920 \div 1130$ simulations (Table VI) that are needed by the hybrid method.

\section{SENSITIVITY ANALYSIS}

The uncertainty in many SAPS variables over which the designer has no control, such as wind speed, future fuel prices, and electric load, makes the need for sensitivity analysis essential. Moreover, in some SAPS components (such as PVs), there is an option of choosing alternative technologies, which present different performance and cost characteristics. In this section, eight alternative scenarios have been developed and analyzed. 
TABLE VII

Optimal Results For EACH SCENARIO OF THE Sensitivity ANALysis

\begin{tabular}{c|c|c|c|c|c|c|c|c|c|c}
\hline Scenario & WTs* & $\begin{array}{c}\text { PVs } \\
\left(\mathrm{kW}_{\mathrm{p}}\right)\end{array}$ & $\begin{array}{c}\text { Dsl } * \\
(\mathrm{~kW})\end{array}$ & $\begin{array}{c}\text { Bio* } \\
(\mathrm{kW})\end{array}$ & $\begin{array}{c}\mathrm{FCs} * \\
(\mathrm{~kW})\end{array}$ & Batteries* & $\begin{array}{c}\text { Converter * } \\
(\mathrm{kW})\end{array}$ & $\begin{array}{c}\text { Dispatch } \\
\text { strategy }\end{array}$ & $\begin{array}{c}\text { COE } \\
(€ / \mathrm{kWh})\end{array}$ & $\begin{array}{c}\text { Total number of } \\
\text { evaluations }(\mathrm{SA}+\mathrm{TS})\end{array}$ \\
\hline 1 & $5(36.6 \%)$ & $28(7.1 \%)$ & $30(16.0 \%)$ & $20(8.5 \%)$ & $0(0 \%)$ & $320(22.7 \%)$ & $78(9.1 \%)$ & LF & 0.179312 & $1220(840+380)$ \\
2 & $6(34.3 \%)$ & $36(7.2 \%)$ & $50(30.8 \%)$ & $5(1.2 \%)$ & $5(3.4 \%)$ & $248(15.1 \%)$ & $87(8.0 \%)$ & LF & 0.229038 & $940(540+400)$ \\
3 & $6(40.6 \%)$ & $30(7.1 \%)$ & $50(21.8 \%)$ & $0(0 \%)$ & $0(0 \%)$ & $312(21.1 \%)$ & $87(9.4 \%)$ & LF & 0.193684 & $858(480+378)$ \\
4 & $6(40.0 \%)$ & $22(5.1 \%)$ & $50(22.6 \%)$ & $5(1.2 \%)$ & $0(0 \%)$ & $344(21.8 \%)$ & $87(9.3 \%)$ & LF & 0.196885 & $980(600+380)$ \\
5 & $5(32.9 \%)$ & $28(8.5 \%)$ & $50(26.0 \%)$ & $5(1.3 \%)$ & $0(0 \%)$ & $392(22.5 \%)$ & $84(8.8 \%)$ & LF & 0.199420 & $793(420+373)$ \\
6 & $6(31.1 \%)$ & $34(6.1 \%)$ & $80(39.5 \%)$ & $0(0 \%)$ & $0(0 \%)$ & $248(14.6 \%)$ & $105(8.7 \%)$ & LF & 0.211251 & $930(570+360)$ \\
7 & $7(43.7 \%)$ & $14(3.0 \%)$ & $5(2.4 \%)$ & $50(24.4 \%)$ & $0(0 \%)$ & $280(18.4 \%)$ & $81(8.1 \%)$ & LF & 0.212400 & $898(510+388)$ \\
8 & $7(33.6 \%)$ & $80(12.3 \%)$ & $10(4.7 \%)$ & $30(13.9 \%)$ & $0(0 \%)$ & $240(24.0 \%)$ & $81(11.5 \%)$ & LF & 0.147881 & $918(540+378)$ \\
Initial (best) & $6(40.5 \%)$ & $32(7.5 \%)$ & $50(22.3 \%)$ & $0(0 \%)$ & $0(0 \%)$ & $304(20.7 \%)$ & $84(9.0 \%)$ & LF & 0.194671 & $1130(750+380)$ \\
\hline
\end{tabular}

* The numbers in parentheses represent the share of each component on COE. For example, the share of the five WTs on COE is $36.6 \%$ for scenario 1 .

All scenarios are based on the case study system of Section V-A and include the following modifications:

1) $10 \%$ increase of wind speed;

2) $10 \%$ decrease of wind speed;

3) $5 \%$ increase of solar radiation;

4) $5 \%$ decrease of solar radiation;

5) installation of a two-axis PV tracking system (additional PV cost: $1000 € / \mathrm{kW}_{p}$ );

6) $20 \%$ increase of electric load;

7) increase of diesel fuel price from $1.3 € / \mathrm{L}$ to $2.0 € / \mathrm{L}$;

8) $50 \%$ cost reduction of renewable energy technologies (WTs and PVs).

Table VII presents the optimal configuration for each one of the above eight scenarios. The numbers into parentheses in the columns $2 \div 8$ of Table VII represent the contribution on COE of each component for a given scenario. In each scenario, the number of TS iterations was kept equal to 20 . The study of Table VII draws the following conclusions: 1) the wind potential (scenario 1) is more significant than the solar potential (scenario 3 ) in order to achieve the minimum SAPS COE, 2) the adoption of a two-axis PV tracking system (scenario 5) does not improve the PV penetration due to its high cost, 3) the significance of the RES technologies lower cost is obvious in comparison to the higher diesel fuel price, and 4) the configurations in all the examined scenarios contain large numbers of WTs and batteries, converters of similar sizes, negligible sizes of FCs, and adoption of LF dispatch strategy.

\section{CONCLUSION}

This paper dealt with the optimal sizing problem of SAPS that contain renewable energy technologies. The solution of this problem includes considerable difficulties due to the large number of design options, and the uncertainty in the values of many important input parameters. Two popular metaheuristic methods, namely, SA and TS, are applied and compared. The following main conclusions were drawn: 1) the solution quality was similar for all examined methods, and 2) the calculation time for all methods was very short compared to the prohibitive time required using the complete enumeration method.

By studying the particular characteristics of each method, SA presents faster convergence in the neighborhood of optimal solutions, whereas TS is more efficient in finding the best solution in a given neighborhood. For this reason, a hybrid methodology was proposed that combines the advantages of each ex- amined metaheuristic method. In this hybrid SA-TS optimization method, the solution obtained from SA was used as the initial (feasible) solution for the TS algorithm. The results showed that the proposed hybrid method improves the solution quality, without increasing significantly the number of required simulations.

In all examined SAPS configurations, the target was their economic and reliable operation throughout the year, with high penetration of renewable technologies and low initial cost. The analysis of results showed the significant contribution of wind turbines and batteries. Methanol fuel cells were not proved currently effective due to their high cost, but in the future their use may be significantly expanded.

\section{REFERENCES}

[1] M. D. Ilić, J.-Y. Joo, L. Xie, M. Prica, and N. Rotering, "A decision-making framework and simulator for sustainable electric energy systems," IEEE Trans. Sustain. Energy, vol. 2, no. 1, pp. 37-49, Jan. 2011.

[2] T. K. A. Brekken, A. Yokochi, A. von Jouanne, Z. Z. Yen, H. Max Hapke, and D. A. Halamy, "Optimal energy storage sizing and control for wind power applications," IEEE Trans. Sustain. Energy, vol. 2, no. 1, pp. 69-77, Jan. 2011

[3] L. A. de Souza Ribeiro, O. R. Saavedra, S. L. de Lima, and J. G. de Matos, "Isolated micro-grids with renewable hybrid generation: The case of Lençóis island," IEEE Trans. Sustain. Energy, vol. 2, no. 1, pp. 1-11, Jan. 2011.

[4] W. Zhou, C. Lou, Z. Li, L. Lu, and H. Yang, "Current status of research on optimum sizing of stand-alone hybrid solar-wind power generation systems," Appl. Energy, vol. 87, pp. 380-389, 2010.

[5] National Renewable Energy Laboratory (NREL): HOMER, The Micropower Optimization Model [Online]. Available: http://www.homerenergy.com

[6] D. Connoly, H. Lund, B. V. Mathiesen, and M. Leahy, "A review of computer tools for analysing the integration of renewable energy into various energy systems," Appl. Energy, vol. 87, pp. 1059-1082, 2010.

[7] Y. A. Katsigiannis, P. S. Georgilakis, and E. S. Karapidakis, "Genetic algorithm solution to optimal sizing problem of small autonomous hybrid power systems," Lecture Notes Artif. Intell., vol. 6040, pp. $327-332,2010$.

[8] E. Koutroulis, D. Kolokotsa, A. Potirakis, and K. Kalaitzakis, "Methodology for optimal sizing of stand-alone photovoltaic/windgenerator systems using genetic algorithms," Solar Energy, vol. 80, pp. 1072-1088, 2006.

[9] Y. A. Katsigiannis, P. S. Georgilakis, and E. S. Karapidakis, "Multiobjective genetic algorithm solution to the optimum economic and environmental performance problem of small autonomous hybrid power systems with renewables," IET Renew. Power Generation, vol. 4, pp. 404-419, 2010.

[10] Y. A. Katsigiannis, P. S. Georgilakis, and E. S. Karapidakis, "A simulated annealing algorithm for optimal sizing of a small isolated power system," in Proc. 6th Japanese-Mediterranean Workshop on Applied Electromagnetic Engineering for Magnetic, Superconducting and Nano Materials (JAPMED'6), Bucharest, Romania, Jul. 2009. 
[11] Y. A. Katsigiannis and P. S. Georgilakis, "Optimal sizing of small isolated hybrid power systems using tabu search," J. Optoelectron. Adv. Mater., vol. 10, pp. 1241-1245, May 2008.

[12] S. M. Hakimi and S. M. Moghaddas-Tafreshi, "Optimal sizing of a stand-alone hybrid power system via particle swarm optimization for Kahnouj area in south-east of Iran," Renew. Energy, vol. 34, pp. $1855-1862,2009$

[13] A. H. Mantawy, Y. L. Abdel-Magid, and S. Z. Selim, "Integrating genetic algorithms, tabu search and simulated annealing for the unit commitment problem," IEEE Trans. Power Syst., vol. 14, no. 3, pp. 829-836, Aug. 1999.

[14] R. A. Gallego, A. J. Monticelli, and R. Romero, "Optimal capacitor placement in radial distribution networks," IEEE Trans. Power Syst., vol. 16, no. 4, pp. 630-637, Nov. 2001.

[15] S. W. Lina and K. C. Ying, "Applying a hybrid simulated annealing and tabu search approach to non-permutation flowshop scheduling problems," Int. J. Prod. Res., vol. 47, pp. 1411-1424, 2009.

[16] T. Lambert, P. Gilman, and P. Lilienthal, "Micropower system modeling with HOMER," in Integration of Alternative Sources of Energy, F. A. Farret and M. G. Simões, Eds. Hoboken, NJ: Wiley, 2006, pp. 379-418.

[17] M. Thomson and D. G. Infield, "Impact of widespread photovoltaics generation on distribution systems," IET Renew. Power Generation, vol. 1, pp. 33-40, 2007.

[18] T. Markvart and L. Castañer, Practical Handbook of Photovoltaics: Fundamentals and Applications. U.K.: Elsevier, 2003.

[19] O. Skarstein and K. Uhlen, "Design considerations with respect to long-term diesel saving in wind/diesel plants," Wind Eng., vol. 13, pp. 72-87, 1989.

[20] A. Demirbas, Biodiesel-A Realistic Fuel Alternative for Diesel. London: Springer-Verlag, 2008.

[21] T. Ruberti, "Off-grid hybrids: Fuel cell solar-PV hybrids," Refocus, vol. 4, no. 5, pp. 54-57, 2003.

[22] C. D. Barley and C. B. Winn, "Optimal dispatch strategy in remote hybrid power systems," Solar Energy, vol. 58, pp. 165-179, 1996.
[23] K. Y. Lee and M. A. El-Sharkawi, Modern Heuristic Optimization Techniques: Theory and Applications to Power Systems. Hoboken, NJ: Wiley, 2008.

Yiannis A. Katsigiannis was born in Athens, Greece, in 1975. He received the Diploma in production engineering and management, the M.S. degree, the Diploma in environmental engineering, and the Ph.D. degree from the Technical University of Crete in 2000, 2003, 2005, and 2008, respectively.

His research areas are artificial intelligence, renewable energy sources, and their integration to power systems.

Dr. Katsigiannis is a member of the Technical Chamber of Greece.

Pavlos S. Georgilakis (SM'11) received the Diploma and Ph.D. degrees in electrical and computer engineering from the National Technical University of Athens (NTUA), Athens, Greece, in 1990 and 2000, respectively.

$\mathrm{He}$ is currently a Lecturer at the School of Electrical and Computer Engineering of NTUA. From 2004 to 2009, he was an Assistant Professor in the Production Engineering and Management Department of the Technical University of Crete, Greece. His current research interests include renewable energy sources, and transmission and distribution of electric power.

Emmanuel S. Karapidakis (M'03) is currently an Assistant Professor in the Technological Educational Institute of Crete (TEIC), Greece.

$\mathrm{He}$ is team leader in the Renewable Energy Engineering Laboratory of TEIC. His research interests include dynamic security, artificial intelligence techniques, dispersed generation, and energy planning.

Dr. Karapidakis is a member Technical Chamber of Greece, and since 2007 he is a team leader of the local "Energy \& New Technologies" policy-making committee. 